\title{
La Idoneidad Didáctica como herramienta metodológica: desarrollo y contextos de uso
}

\author{
Omar Malet \\ Belén Giacomone \\ Ana María Repetto
}

\begin{abstract}
Resumen: El objetivo de esta investigación es contribuir al conocimiento del estado de desarrollo de la Idoneidad Didáctica, una de las herramientas teórico-metodológicas del Enfoque Ontosemiótico del Conocimiento y la Instrucción Matemáticos. Se trata de una investigación cualitativa, basada en el análisis de contenido de 85 trabajos publicados entre 2005 y 2020, en español, portugués e inglés. Como resultado del análisis, se identificaron nueve categorías emergentes que reflejan los distintos usos de la Idoneidad Didáctica como herramienta metodológica en las investigaciones sobre educación. Las dos categorías que concentran más trabajos son la Idoneidad como referencia para valorar un proceso de estudio y la que implica el desarrollo de la competencia de reflexión didáctica en la formación docente. Los resultados reflejan las tendencias en la agenda científica y eventuales líneas de investigación pendientes.
\end{abstract}

Palabras clave: Educación matemática. Idoneidad didáctica. Estado del arte. Enfoque Ontosemiótico

Omar Malet por la Universidad Nacional de Cuyo, República Argentina. Profesor de la Universidad Nacional de Tres de Febrero, Caseros, provincia de Buenos Aires, República Argentina https://orcid.org/0000-0003-4112-9217 $\triangle$ omalet@untref.edu.ar

Belén Giacomone Doctora en Ciencias de la Educación por la Universidad de Granada, España. Profesora asociada de la Universidad de San Marino, República de San Marino.

https://orcid.org/0000-0001-6752-2362

$\square$ belen.giacomone@unirsm.sm

Ana María Repetto Doctora en Ciencias de la Educación por la Universidad Nacional de Cuyo,

República Argentina. Profesora titular de la Universidad Nacional de Cuyo Mendoza, República Argentina. https://orcid.org/0000-0002-3446-3500 $\bowtie$ anarepetto@fed.uncu.edu.ar

Recebido em 10/05/2021

Aceito em 24/05/2021

Publicado em 22/06/2021

\section{A Idoneidade didática como ferramenta metodológica: desenvolvimento e contextos de uso}

Resumo: 0 objetivo desta pesquisa é contribuir para o conhecimento do estado de desenvolvimento da Idoneidade Didática, uma das ferramentas teóricometodológicas da Abordagem Ontosemiótica do Conhecimento e Instrução Matemática. Trata-se de uma pesquisa qualitativa, com base na análise de conteúdo de 85 artigos publicados entre 2005 e 2020, nos idiomas espanhol, português e inglês. Como resultado da análise, foram identificadas nove categorias emergentes que refletem os diferentes usos da Idoneidade Didática como ferramenta metodológica na pesquisa em educação. As duas categorias que mais concentram os trabalhos são a Idoneidade como referência para avaliar um processo de estudo e aquela que implica o desenvolvimento da competência de reflexão didática na formação de professores. Os resultados refletem as tendências da agenda científica e eventuais vagas.

Palavras-chave: Educação matemática. Idoneidade didática. Estado da arte. Abordagem ontosemiótica.

\section{Didactic Suitability as a methodological tool: development and contexts of use}

Abstract: This research is aimed at contributing to the knowledge of the state of development of Didactic Suitability, one of theoretical-methodological tools of the Onto-Semiotic Approach to Mathematical Knowledge and Instruction. The research has a qualitative approach based on the content analysis of 85 papers published between 2005 and 2020, in Spanish, Portuguese, and English. As a result of the analysis, nine emerging categories were identified that reflect the different uses of Didactic Suitability as a methodological tool in research on education. The categories that concentrate the most papers are two: Didactic Suitability as a reference to assess a study process, and one that implies the development of 
didactic reflection competence in teacher education. The results show the trends in the scientific agenda and eventual vacancies.

Keywords: Mathematics education. Didactic suitability. State of the art. Onto-semiotic Approach

\section{Introducción}

El objetivo de este trabajo es indagar en las distintas investigaciones que se vienen llevando a cabo sobre el desarrollo y los contextos de uso de la Idoneidad Didáctica a lo largo del tiempo, siendo esta una herramienta teórico-metodológica específica que permite abordar cuestiones claves de la investigación didáctica (GODINO, 2002). Pero también, se trata de una contribución al estado del arte que permita crear nuevas miradas de investigación y plasmar futuras líneas de investigación.

En efecto, el estado del arte es un paso clave en la etapa preliminar de una investigación siendo el objetivo principal analizar la bibliografía existente sobre un determinado tópico. Los resultados dan cuenta de los distintos métodos y enfoques teóricos que se utilizan y que surgen del estudio del mismo, y, a la vez, permiten al investigador clarificar nuevos interrogantes para delimitar su problema de investigación. Pero también, el estado del arte es considerado una investigación con desarrollo autónomo en el campo de la investigación documental (HOYOS BOTERO, 2000) que pretende "dar cuenta de construcciones de sentido sobre datos que apoyan un diagnóstico y un pronóstico en relación con el material documental sometido a análisis" (p. 57).

Este trabajo se ha organizado en cinco secciones. En la Sección 2, se describe el enfoque teórico en el cual se enmarca la Idoneidad Didáctica y el problema específico de investigación. En la Sección 3, se describen la metodología empleada, los métodos de búsqueda y la descripción del material empírico. En la Sección 4, y sin pretensiones de exhaustividad, se describen los resultados del análisis de la muestra. Finalmente, en la Sección 5 , se presentan las conclusiones reflejando la progresiva expansión de los contextos de uso de la Idoneidad Didáctica, las tendencias que se manifiestan en esa expansión y líneas de investigación pendientes.

\section{Fundamentos teóricos y problema específico de investigación}

En el marco del Enfoque Ontosemiótico del Conocimiento y la Instrucción Matemáticos (EOS), enfoque desarrollado por Godino y colaboradores (GODINO, 2002; GODINO, BATANERO y FONT, 2007; 2020), la idoneidad didáctica de un proceso de estudio se define como 
circunstancias y recursos disponibles (entorno). Esto supone la articulación coherente y sistémica de seis facetas: epistémica, ecológica, cognitiva, afectiva, interaccional y mediacional. (GODINO, BATANERO y FONT, 2020, p. 11)

Con el propósito de dar juicios valorativos o razonados sobre la idoneidad de un determinado proceso de estudio, en el marco del EOS se desarrolló la noción de Idoneidad Didáctica (GODINO, 2013) como herramienta teórico-metodológica. Esta noción puede servir "como punto de partida para una teoría de diseño instruccional que tenga en cuenta, de manera sistémica, las dimensiones [facetas] epistémica-ecológica, cognitiva-afectiva, interaccionalmediacional implicadas en los procesos de estudio de las áreas curriculares específicas" (GODINO, 2013, p. 115). En este sentido, puede aplicarse para analizar distintos aspectos de dicho proceso, como, por ejemplo, al análisis de la planificación o implementación de una secuencia didáctica, de una sesión de clase, o de un curso completo, al análisis de programas curriculares, libros de texto, producciones de los estudiantes, entre otros aspectos tanto parciales como globales.

Para facilitar la reflexión, global o parcial, sobre un determinado proceso de estudio, con la noción de la Idoneidad Didáctica se pretende valorar las seis facetas que intervienen en el diseño, implementación y evaluación de procesos de enseñanza y aprendizaje, y las relaciones que se establecen entre las mismas (Figura 1).

Figura 1. Facetas de un proceso instruccional

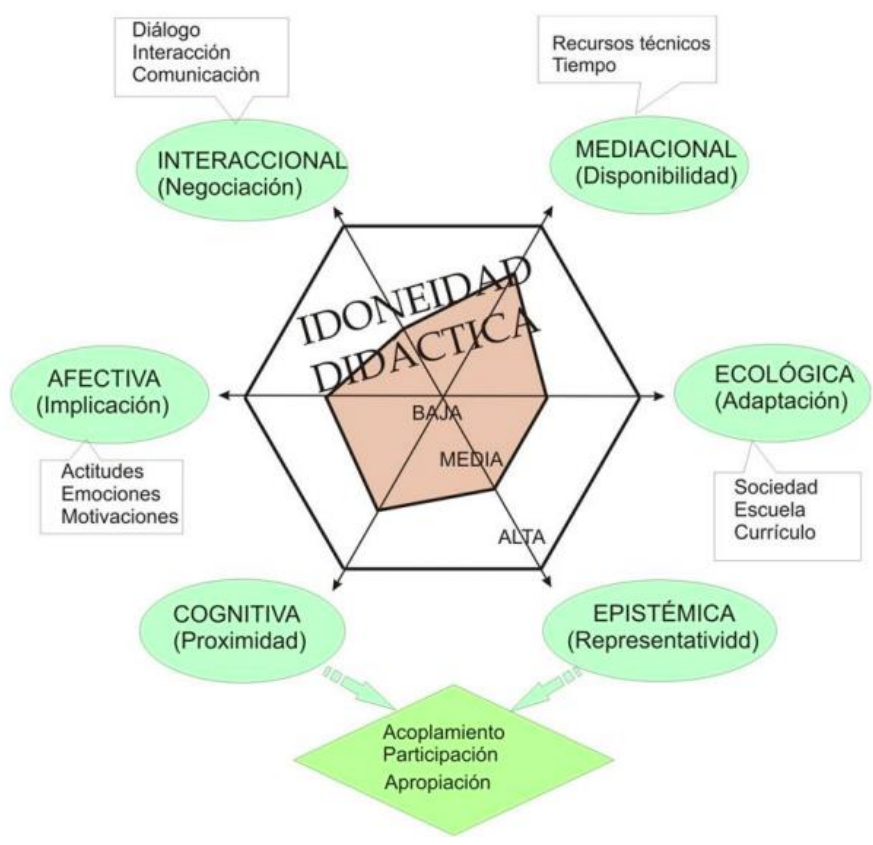

Fuente: Godino (2013, p. 116) 
Para valorar y mejorar cada fase de un proceso instruccional, el EOS aporta un sistema de criterios e indicadores empíricos para cada una de las facetas señaladas en la Figura 1, y para la articulación de las mismas. Por ejemplo, la fase de diseño de un proceso de instrucción requiere que el profesor-investigador realice a priori una reconstrucción del significado global de referencia del contenido que se pretende enseñar (GODINO, BURGOS y GEA, 2021) y la adapte al contexto en el cual está enseñando. Esto es fundamental para lograr una alta idoneidad epistémicoecológica.

Los criterios de valoración o criterios de idoneidad didáctica "se han confeccionado teniendo en cuenta las tendencias, los principios y los resultados de la investigación en el área de Didáctica de las Matemáticas" (BREDA, FONT y PINO-FAN, 2018, p. 271). Tales criterios son generales y se encuentran detalladamente en el artículo de Godino (2013).

Simultáneamente con la difusión del EOS a nivel internacional, la noción de Idoneidad Didáctica ha suscitado un interés creciente entre los investigadores y los docentes, sin duda motivado por su versatilidad como herramienta teórica y metodológica.

En particular, la herramienta ha generado un interés especial en el campo de la formación de profesores. En el marco del EOS, su conocimiento y uso competente ha sido incorporado al Modelo de Conocimientos y Competencias Didáctico-Matemáticas (CCDM) del profesor de matemáticas (GODINO et al., 2017). Bajo este enfoque, se la considera como una de las competencias específicas que debería desarrollar el profesor en su formación inicial y continua: la competencia de análisis y valoración de la idoneidad didáctica, que supone el conocimiento de la herramienta y su uso competente.

Cobra sentido preguntarse, entonces, cuál ha sido el desarrollo de la noción desde el punto de vista de sus aplicaciones y contextos de uso, desde el primer trabajo sobre la temática, publicado en 2005, hasta la actualidad (2020). Para dar respuesta a tales interrogantes, en este estudio se han analizado 85 investigaciones en las que la Idoneidad Didáctica fue aplicada como herramienta metodológica.

\section{Metodología}

La investigación se inscribe en el paradigma interpretativo y como tal es de tipo cualitativo (VASILACHIS DE GIALDINO, 2006). La técnica utilizada es el análisis de contenido (BARDIN, 1996) y fue aplicada a una muestra de investigaciones seleccionada y recolectada a partir de la búsqueda de producciones científicas. 


\subsection{Método de búsqueda}

La recolección del material empírico se centró en artículos de revistas científicas y capítulos de libros online, incluyendo actas de congresos, publicados entre 2005 y 2020, dando como resultado 85 trabajos relevantes con revisión por pares. Se accedió a los textos disponibles para su lectura, revisión y copiado textual de la información requerida según categorías analíticas que fueron determinadas a priori.

La búsqueda se realizó en distintos grupos de bases de datos: el primero, formado por Scopus, Springer, Web of Science, Redalyc y Scielo; el segundo, el repositorio de la Universidad de Granada http://enfoqueontosemiotico.ugr.es/pages/idoneidad.html.

Dado el alcance internacional del objeto de investigación, las palabras clave de búsqueda utilizadas en todos los medios fueron: idoneidad didáctica, adequação didática, idoneidade didática, didactic suitability, aptitude didactique.

Es importante destacar que la búsqueda arrojó como resultado numerosas Tesis Doctorales y de Fin de Máster cuyo análisis no es abordado en este estudio. Para dar cuenta del número real de tesis, se requiere realizar otro tipo de estudio que implicaría tener en cuenta buscadores específicos tales como Dialnet-tesis doctorales o REDIAL-TESIS, entre otros, dado que no todas las tesis están disponibles en las bases consultadas. Por ejemplo, en Font (2011) se describe el completo proceso de formación inicial en un máster de Profesor de Secundaria de Matemáticas de la Universitat de Barcelona en el cual se utiliza la Idoneidad Didáctica para el Trabajo de Fin de Máster. No obstante, muchas de las tesis que se obtuvieron como resultado directo de la búsqueda (por ejemplo: BELTRÁN-PELLICER, 2016; BREDA, 2016; BURGOS, 2020; CRISÓSTOMO, 2012; GIACOMONE, 2018; RIVAS, 2014) dieron pie a una o más publicaciones en revistas científicas o capítulos de libros (entre ellos, actas de congresos) que figuran entre las recolectadas en esta búsqueda, ratificando una tendencia consolidada en el ámbito académico.

\subsection{Descripción de la muestra}

La muestra está formada por 85 trabajos, de los cuales 58 proceden de 39 revistas y 27 de 15 capítulos de libros. No se consideraron trabajos in print. En la Tabla 1 se presenta la cantidad de trabajos publicados en cada medio. El total de trabajos por período de tiempo permite analizar el ritmo de cambio de la producción científica sobre la temática. 
Tabla 1: Listado de publicaciones científicas y cantidad de trabajos publicados

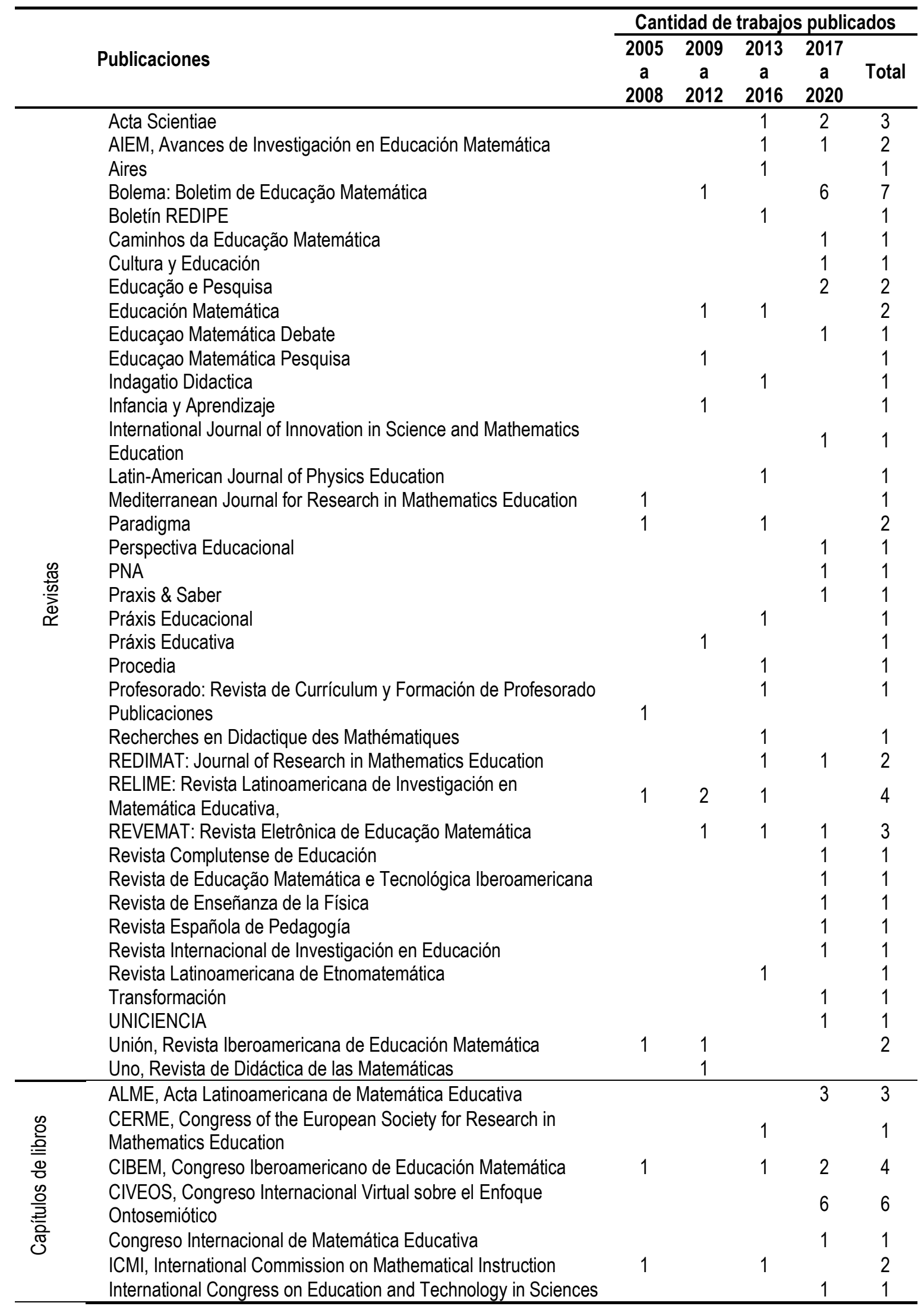




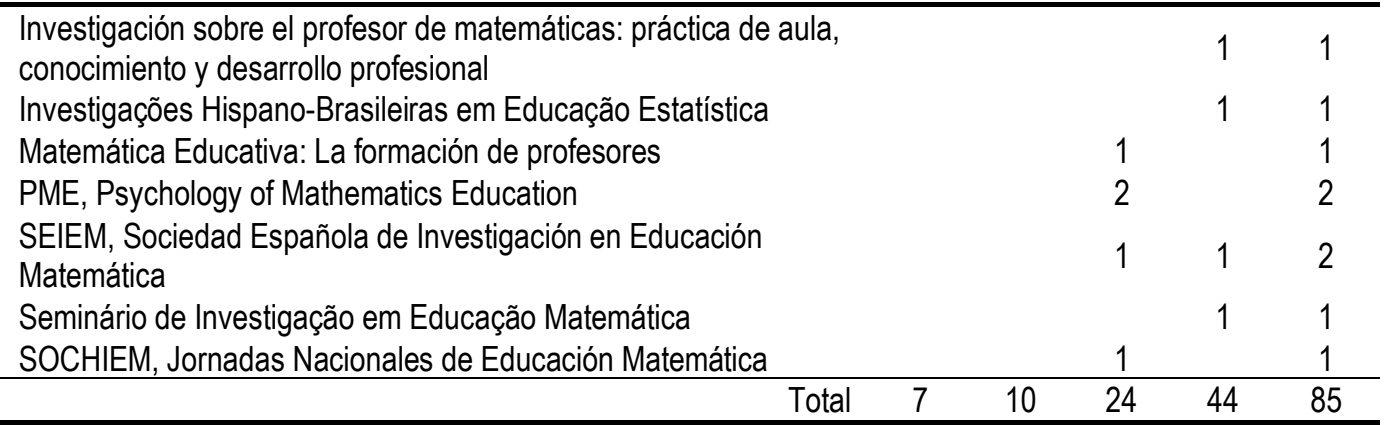

Fuente: Elaboración propia (2021).

\subsection{Categorías de análisis}

El foco del estudio está puesto en la identificación -mediante el análisis de contenido- de categorías emergentes que permitan clasificar los distintos contextos de uso de la Idoneidad Didáctica y la finalidad de la investigación. Sin embargo, con el doble propósito de contribuir a una caracterización más precisa de aquellos contextos, y de reportar la falta de estudios en algunas áreas, también se relevó información en función de algunas categorías analíticas definidas a priori y consensuadas por los autores: el nivel educativo al que concierne cada investigación (educación infantil, primaria, secundaria, universitaria, formación docente inicial o continua), el contenido involucrado (contenido matemático, de las ciencias experimentales, didáctico), los actores que intervienen en la aplicación del constructo (investigadores, observadores, docentes, estudiantes, individuos, grupos), el proceso de estudio investigado (ciclos educativos, programas de estudio, videos, libros de texto, acciones didácticas, producciones escritas, valoraciones profesorales, actividades y secuencias didácticas) y las facetas de la idoneidad didáctica implicadas (epistémica, ecológica, cognitiva, afectiva, interaccional y mediacional). Se registró también el lugar de edición de la publicación. Por razones de espacio, en este artículo no se reportan detalladamente los resultados derivados de tales categorías.

En cuanto a las categorías emergentes, que se enumeran en la sección siguiente, fueron generadas inductiva y recursivamente por un proceso de verificación cruzada entre los tres autores, es decir, por triangulación de investigadores (PATTON, 2002), hasta alcanzar el punto de saturación, esto es, hasta que las categorías identificadas permitieron encuadrar satisfactoriamente los 85 trabajos. Esta técnica permite contrastar y recuperar (si es que existen) puntos de vista diferentes que colaboren en lograr una comprensión más acabada del objeto de estudio. Al mismo tiempo, se configura como instrumento de validación que imprime base científica al procedimiento de investigación cualitativa llevado a cabo. 


\section{Resultados}

Un primer resultado del estudio se manifiesta en la cronología de la Tabla 1 donde se aprecia un crecimiento notable y sostenido en el número de trabajos publicados a lo largo del tiempo. En efecto, mientras que en el cuatrienio 2005-2008 se dieron a conocer solo 7 investigaciones, en el cuatrienio siguiente, 2009-2012, se difundieron 10, en el cuatrienio 20132016, 24, y en el último cuatrienio, 2017-2020, 44.

A este aumento considerable se lo puede interpretar como un creciente interés de la comunidad científica por comprender y aplicar la Idoneidad Didáctica en el campo de la educación, no solo en el área de Matemática sino también en el de las Ciencias Experimentales. Por ejemplo, en el 2015, una investigación aplica la herramienta para valorar un proceso de enseñanza de la Física, y en 2017, se la utiliza para determinar criterios de idoneidad sobre el uso de experimentos de acceso remoto en la enseñanza universitaria de la Física en la modalidad a distancia.

A continuación, se detallan las 85 investigaciones que conforman la muestra de material empírico, ordenadas según el año de publicación.

1. GODINO, Juan D.; WILHELMI, Miguel R.; BENCOMO, Delisa. Suitability criteria of a mathematical instruction process. A teaching experience of the function notion. Mediterranean journal for research in mathematics education, v. 4, n. 2, p. 1-26, 2005.

2. GODINO, Juan D.; BENCOMO, Delisa; FONT, Vicenç; WILHELMI, Miguel R. Análisis y valoración de la idoneidad didáctica de procesos de estudio de las Matemáticas. Paradigma, v. 27, n. 2, p. 221-252, 2006.

3. CASTRO HERNÁNDEZ, Carlos. La evaluación de métodos para la enseñanza y el aprendizaje de las Matemáticas en la educación infantil. Unión, n. 11, p. 59-77, 2007.

4. GODINO, Juan D.; BATANERO, Carmen. Formación de profesores de Matemáticas basada en la reflexión guiada sobre la práctica. In: Conferencia Invitada al VI CIBEM, Puerto Montt (Chile), 4-9. Enero, 2008. Disponible en https://www.ugr.es/ jgodino/eos/fprofesores_reflexion_guiada_22dic08.pdf

5. GODINO, Juan D.; BATANERO, Carmen; ROA, Rafael; WILHELMI, Miguel R. Assessing and developing pedagogical content and statistical knowledge of primary school teachers through project work. In: BATANERO, Carmen; BURRILL, Gail; READING, Chris; ROSSMAN, Allan (Eds.). Joint ICMI/IASE Study: Teaching Statistics in school Mathematics. Challenges for teaching and teacher education. Proceedings of the ICMI Study 18 and 2008 IASE Round Table Conference. Monterrey: IASE, 2008.

6. GODINO, Juan D.; FONT, Vicenç; WILHELMI, Miguel R. Análisis didáctico de procesos de estudio matemático basado en el enfoque ontosemiótico. Publicaciones, n. 38, p. 25-49, 2008. 
7. RAMOS, Ana B.; FONT, Vicenç. Criterios de idoneidad y valoración de cambios en el proceso de instrucción matemática. RELIME: Revista Latinoamericana de Investigación en Matemática Educativa, v. 11, n. 2, 233-265, 2008.

8. ALSINA, Ángel; DOMINGO, Marta. Idoneidad didáctica de un protocolo sociocultural de enseñanza y aprendizaje de las Matemáticas. RELIME: Revista Latinoamericana de Investigación en Matemática Educativa, v. 13, n. 1, p. 7-32, 2010.

9. FONT, Vicenç; PLANAS, Nuria; GODINO, Juan D. Modelo para el análisis didáctico en Educación Matemática. Infancia y Aprendizaje, v. 33, n. 1, p. 89-105, 2010.

10. FONT, Vicenç. Competencias profesionales en la formación inicial de profesores de Matemáticas de secundaria. Unión, n. 26, p. 9-25, 2011.

11. POCHULU, Marcel; FONT, Vicenç. Análisis del funcionamiento de una clase de Matemáticas no significativa. RELIME: Revista Latinoamericana de Investigación en Matemática Educativa, v. 14, n. 3 , p. 361-394, 2011.

12. ARTEAGa, Pedro; batanero, Carmen; CañadAS, Gustavo; GeA María M. Evaluación del conocimiento especializado de la Estadística en futuros profesores mediante el análisis de un proyecto estadístico. Educação Matemática Pesquisa, v. 14, n. 2, p. 279-297, 2012.

13. CONTRERAS DE LA FUENTE, Ángel; GARCÍA ARMENTEROS, Manuel; FONT, Vicenç. Análisis de un proceso de estudio sobre la enseñanza del límite de una función. Bolema: Boletim de Educação Matemática, v. 26, n. 42B, p. 667-690, 2012.

14. GIMÉNEZ, Joaquim; VANEGAS, Yuly M.; FONT, Vicenç; FERRERES, Susanna V. El papel del trabajo final de Máster en la formación del profesorado de Matemáticas. Uno, Revista de Didáctica de las Matemáticas, n. 61, p. 76-86, 2012.

15. GODINO, Juan D.; RIVAS, Hernán; ARTEAGA, Pedro; (2012). Inferencia de indicadores de idoneidad didáctica a partir de orientaciones curriculares. Práxis Educativa, v. 7, n. 2, p. 331-354.

16. GODINO, Juan D.; RIVAS, Mauro; CASTRO, Walter F.; KONIC, Patricia. Desarrollo de competencias para el análisis didáctico del profesor de Matemáticas. REVEMAT, Revista Eletrônica de Educação Matemática, v. 7, n. 2, p. 1-21, 2012.

17. ROBLES ARREDONDO, Martha Gabriela; DEL CASTILLO BOJÓRQUEZ, Ana Guadalupe; FONT, Vicenç. Análisis y valoración de un proceso de instrucción de la derivada. Educación Matemática, v. 24, n. 1, p. 5-41, 2012.

18. CASTRO, Andreia de; SANTANA, Filipa; NETO, Teresa; ÓRFÃO, Isabel. Iniciação à investigação em Educação Matemática: Exemplo de duas tarefas com recurso ao Geogebra. Indagatio Didactica, v. 5, n. 1, p. 127-148, 2013.

19. FONT, Vicenç; ADÁN, Marta. Valoración de la idoneidad matemática de tareas. In: BERCIANO ALCARAZ, Ainhoa; GUTIÉRREZ PEREDA, Guadalupe; ESTEPA CASTRO, Antonio; CLIMENT, Nuria R. (Eds.). Investigación en Educación Matemática XVII. Bilbao: SEIEM, 2013, p. 283-291. 
20. GIMÉNEZ, Joaquim; FONT, Vicenç; VANEGAS, Yuly M. Designing professional tasks for didactical analysis as a research process. In: MARGOLINAS, Claire (Ed.). Task design in Mathematics Education. Proceedings of ICMI Study 22. Oxford: Hal, 2013.

21. GODINO, Juan D.; BATANeRO, Carmen; RIVAS, Hernán; ARTEAgA, Pedro. Componentes e indicadores de idoneidad de programas de formación de profesores en Didáctica de las Matemáticas. REVEMAT, Revista Eletrônica de Educação Matemática, v. 8, n. 1, p. 46-74, 2013.

22. PINO-FAN, Luis R.; CASTRO, Walter F.; GODINO, Juan D.; FONT, Vicenç. (2013). Idoneidad epistémica del significado de la derivada en el currículo de bachillerato. Paradigma, v. 34, n. 2, p. 123150.

23. VALERA HERRERA, Erika Gabriela; MARTíNEZ DE LÓPEZ, Angélica María. La circunferencia y el círculo en educación primaria: Una propuesta desde la idoneidad cognitiva, mediacional y ecológica. In: SEMUR, Sociedad de Educación Matemática Uruguaya (Ed.). VII CIBEM, Congreso lberoamericano de Educación Matemática. Montevideo: SEMUR, 2013.

24. ARTEAGA, Pedro; CONTRERAS, José Miguel; CAÑADAS, Gustavo. Conocimiento de la Estadística y Ios estudiantes en futuros profesores: Un estudio exploratorio. AIEM, n. 6, p. 63-84, 2014.

25. CASTRO GORDILLO, Walter Fernando; VELÁSQUEZ ECHAVARRÍA, Hilduara. Idoneidad didáctica de la práctica de maestros en formación inicial en un contexto urbano de conflicto social violento. Revista Latinoamericana de Etnomatemática, v. 7, n. 3, p. 33-54, 2014.

26. GODINO, Juan D.; RIVAS, Hernán; ARTEAGA, Pedro; LASA, Aitzol; WILHELMI, Miguel R. Ingeniería didáctica basada en el Enfoque ontológico-semiótico del conocimiento y la instrucción matemáticos. Recherches en Didactique des Mathématiques, v. 34, n. 2/3, p. 167-200, 2014.

27. LARIOS OSORIO, Victor; FONT, Vicenç. El estudio de la práctica docente para un diseño de formación para profesores de Matemáticas. In: DOLORES FLORES, Crisólogo; GARCÍA GONZÁLEZ, María del Socorro; HERNÁNDEZ SÁNCHEZ, Judith Alejandra; SOSA GUERRERO, Leticia (Eds.). Matemática Educativa: La formación de profesores. México: Díaz de Santos, 2014, p. 223-239.

28. ROBLES ARREDONDO, Martha Gabriela; TELLECHEA ARMENTA, Eduardo; FONT, Vicenç. Una propuesta de acercamiento alternativo al teorema fundamental del Cálculo. Educación Matemática, v. 26, n. 2, p. 69-109, 2014.

29. VANEGAS, Yuly M.; GIMÉNEZ, Joaquim; FONT, Vicenç; DÍEZ-PALOMAR, Javier. Improving reflective analysis of a secondary school Mathematics teachers' program. In: NICOL, Cynthia; OESTERLE, Susan; LILJEDAHL, Peter; ALLAN, Darien (Eds.). Proceedings of the Joint Meeting of PME 38 and PME-NA 36. Vancouver: PME, 2014, v. 5, p. 321-328.

30. FERRERES, Susana.; VANEGAS, Yuly M. Uso de criterios de calidad en la reflexión sobre la práctica de los futuros profesores de secundaria de Matemáticas. Procedia, n. 196, p. 219-225, 2015.

31. GODINO, Juan D. La idoneidad didáctica como herramienta de análisis y reflexión sobre la práctica del profesor de Matemáticas. In: VÁSQUEZ, Claudia; RIVAS, Hernán; PINCHEIRA, Nataly; ROJAS, 
Francisco; SOLAR, Horacio; CHANDIA, Eugenio M.; PARRAGUEZ, Marcela (Eds.). Jornadas Nacionales de Educación Matemática XIX (pp. 32-41). Villarrica: SOCHIEM, 2015.

32. PARRA BERMÚDEZ, Francisco Javier; ÁVILA GODOY, Ramiro. Hacia una idoneidad didáctica en una clase de Física. Latin-American Journal of Physics Education, v. 9, n. S1, p. 205-1-205-7, 2015.

33. SECKEL, María José; FONT, Vicenç. Competencia de reflexión en la formación inicial de profesores de Matemática en Chile. Práxis Educacional, v. 11, n. 19, p. 55-75, 2015.

34. VANEGAS, Yuly M.; FONT, Vicenç; GIMÉNEZ, Joaquim. How future teachers improve epistemic quality of their own mathematical practices. Proceedings of the 9th Congress of the European Society for Research in Mathematics Education. Prague: CERME, 2015.

35. AROZA, Carlos; GODINO, Juan D.; BELTRÁN-PELLICER, Pablo. Iniciación a la innovación e investigación educativa mediante el análisis de la idoneidad didáctica de una experiencia de enseñanza sobre proporcionalidad. Aires, v. 6, n. 1, 2016.

36. BREDA, Adriana; LIMA, V. M. R. Estudio de caso sobre el análisis didáctico realizado en un trabajo final de un máster para profesores de Matemáticas en servicio. REDIMAT: Journal of Research in Mathematics Education, v. 5, n. 1, p. 74-103, 2016.

37. BREDA, Adriana; PINO-FAN, Luis R.; FONT, Vicenç. Establishing criteria for teachers' reflection on their own practices. In: CSÍKOS, Csaba; RAUSCH, Attila; SZITÁNYI, Judit (Eds.). Proceedings of the 40th Conference of the International Group for the Psychology of Mathematics. Szeged: PME, 2016, v. 1, p. 283.

38. DÍAZ-LEVICOY, Danilo; GIACOMONE, Belén; LÓPEZ-MARTíN, María del Mar; PIÑEIRO, Juan Luis. Estudio sobre los gráficos estadísticos en libros de texto digitales de educación primaria española. Profesorado, Revista de Currículum y Formación de Profesorado, v. 20, n. 1, p.133-156, 2016.

39. GÓMEZ BLANCO, Wilmer Merardo; TOVAR ESPINEL, Sandra Jazmín; RAMÍREZ VANEGAS, Guillermo Alfonso. La teoría de la idoneidad didáctica: Una posible herramienta para analizar prácticas pedagógicas en Matemáticas. Boletín REDIPE, v. 5, n. 10, p. 92-101, 2016.

40. POCHULU, Marcel; FONT, Vicenç; RODRÍGUEZ, Mabel. Desarrollo de la competencia en análisis didáctico de formadores de futuros profesores de Matemática a través del diseño de tareas. RELIME: Revista Latinoamericana de Investigación en Matemática Educativa, v. 19, n. 1, p. 71-98, 2016.

41. SOARES, Maria Elaine Santos; KAIBER, Carmen Teresa. Conhecimentos didático-matemáticos mobilizados por professores dos anos iniciais: Uma análise sob a perspectiva do Enfoque ontosemiótico. Acta Scientiae, v. 18, n. 2, p. 435-455, 2016.

42. ARGUEDAS-MATARRITA, Carlos; CONCARI, Sonia Beatriz; GIACOMONE, Belén. La idoneidad didáctica de los laboratorios remotos como recursos para la enseñanza y aprendizaje de la Física. Revista de Enseñanza de la Física, n. 29, p. 511-517, 2017. 
43. ARTEAGA, Pedro., BATANERO, Carmen; GEA María M. La componente mediacional del conocimiento didáctico-matemático de futuros profesores sobre Estadística: Un estudio de evaluación exploratorio. Educaçao Matemática Debate, v. 1, n. 1, p. 54-75, 2017.

44. BELTRÁN-PELLICER, Pablo; GODINO, Juan D. Aplicación de indicadores de idoneidad afectiva en un proceso de enseñanza de Probabilidad en educación secundaria. Perspectiva Educacional, v. 56, n. 2, p. 92-116, 2017.

45. BLANCO-ÁLVAREZ, Hilbert; FERNÁNDEZ-OLIVERAS, Alicia; OLIVERAS, María Luisa. Evaluación de una clase de Matemáticas diseñada desde la Etnomatemática. In: CONTRERAS, José Miguel; ARTEAGA, Pedro; CAÑADAS, Gustavo R.; GEA, María Magdalena; GIACOMONE, Belén; LÓPEZMARTíN, María del Mar (Eds.). Actas del Segundo Congreso Internacional Virtual sobre el Enfoque ontosemiótico del conocimiento y la instrucción matemáticos. Granada: CIVEOS, 2017. Disponible en http://enfoqueontosemiotico.ugr.es/civeos.html

46. CRISOSTOMO DOS SANTOS, Edson Crisostomo; GODINO, Juan D. Conhecimento profissional manifestado por professores-formadores sobre a idoneidade didática do processo de estudo do cálculo integral. In: CONTRERAS, José Miguel; ARTEAGA, Pedro; CAÑADAS, Gustavo R.; GEA, María Magdalena; GIACOMONE, Belén; LÓPEZ-MARTíN, María del Mar (Eds.). Actas del Segundo Congreso Internacional Virtual sobre el Enfoque ontosemiótico del conocimiento y la instrucción matemáticos. Granada: CIVEOS, 2017. Disponible en http://enfoqueontosemiotico.ugr.es/civeos.html

47. CRUZ, Andrea; GEA María M.; GIACOMONE, Belén. Criterios de idoneidad epistémica para el estudio de la Geometría espacial en educación primaria. In: CONTRERAS, José Miguel; ARTEAGA, Pedro; CAÑADAS, Gustavo R.; GEA, María Magdalena; GIACOMONE, Belén; LÓPEZ-MARTíN, María del Mar (Eds.). Actas del Segundo Congreso Internacional Virtual sobre el Enfoque ontosemiótico del conocimiento y la instrucción matemáticos. Granada: CIVEOS, 2017. Disponible en http://enfoqueontosemiotico.ugr.es/civeos.html

48. CRUZ, Andrea; GEA María M.; GIACOMONE, Belén; GODINO, Juan D. Criterios de idoneidad cognitiva para el estudio de la Geometría espacial en educación primaria. In: Federación Española de Sociedades de Profesores de Matemáticas (Ed.). VIII CIBEM, Congreso Iberoamericano de Educación Matemática. Madrid: FESPM, 2017, p. 29-37.

49. Moreira, Celma Bento; Gusmão, Tânia Cristina Silva; FONT, Vicenç. Tarefas matemáticas para a educação infantil: Desenho e avaliação por meio dos critérios de idoneidade didática. In: CONTRERAS, José Miguel; ARTEAGA, Pedro; CAÑADAS, Gustavo R.; GEA, María Magdalena; GIACOMONE, Belén; LÓPEZ-MARTíN, María del Mar (Eds.). Actas del Segundo Congreso Internacional Virtual sobre el Enfoque ontosemiótico del conocimiento y la instrucción matemáticos. Granada: CIVEOS, 2017. Disponible en http://enfoqueontosemiotico.ugr.es/civeos.html

50. NINOW, Valmir; KAIBER, Carmen. Uma análise do conceito de função sob a perspectiva da idoneidade epistêmica do Enfoque ontosemiótico. In: CONTRERAS, José Miguel; ARTEAGA, Pedro; CAÑADAS, Gustavo R.; GEA, María Magdalena; GIACOMONE, Belén; LÓPEZ-MARTíN, María del Mar (Eds.), Actas del Segundo Congreso Internacional Virtual sobre el Enfoque ontosemiótico del conocimiento y 
la instrucción matemáticos. Granada: CIVEOS, 2017. Disponible en http://enfoqueontosemiotico.ugr.es/civeos.html

51. NOGUEIRA, Isabel Cláudia; FERNÁNDEZ-BLANCO, Teresa. Componentes e indicadores de idoneidade didática para procesos de estudo sobre grandezas e sua medida e sua aplicação no ensino básico. In: CONTRERAS, José Miguel; ARTEAGA, Pedro; CAÑADAS, Gustavo R.; GEA, María Magdalena; GIACOMONE, Belén; LÓPEZ-MARTíN, María del Mar (Eds.). Actas del Segundo Congreso Internacional Virtual sobre el Enfoque ontosemiótico del conocimiento y la instrucción matemáticos. Granada: CIVEOS, 2017. Disponible en http://enfoqueontosemiotico.ugr.es/civeos.html

52. NOGUEIRA, Isabel Cláudia; NETO, Teresa. Indicadores de idoneidade didática em contexto de formação inicial de professores: $O$ caso da Ana. Atas do XXVIII Seminário de Investigação em Educação Matemática (pp.142-153). Viseau: APM, 2017.

53. SILVA FERNANDEZ da, José Antonio; PIETROPAOLO, Ruy César. Estudo de componentes e indicadores de idoneidade didática de um curso de formação inicial de professores de Matemática numa instituição brasileira. In: FESPM, Federación Española de Sociedades de Profesores de Matemáticas (Ed.). VIII CIBEM, Congreso Iberoamericano de Educación Matemática. Madrid: FESPM, 2017, p. 460-467.

54. BELTRÁN-PELLICER, Pablo; GIACOMONE, Belén. Developing the competence of didactic suitability analysis and assessment in a postgraduate course through the discussion about the suitability of a teaching experience. REDIMAT: Journal of Research in Mathematics Education, v. 7, n. 2, p. 111-133, 2018.

55. BELTRÁN-PELLICER, Pablo; GIACOMONE, Belén; BURGOS, María. Online educational videos according to specific didactics: The case of Mathematics. Cultura y Educación, v. 30, n. 4, p. 633-662, 2018.

56. BELTRÁN-PELLICER, Pablo; GODINO, Juan D.; GIACOMONE, Belén. Elaboración de indicadores específicos de idoneidad didáctica en Probabilidad: Aplicación para la reflexión sobre la práctica docente. Bolema: Boletim de Educação Matemática, v. 32, n. 61, p. 526-548, 2018.

57. BELTRÁN-PELLICER, Pablo; MEDINA, Antonio; QUERO, Mercedes. Movies and TV series fragments in Mathematics: Epistemic suitability of instructional designs. International Journal of Innovation in Science and Mathematics Education, v. 26, n. 1, p. 16-26, 2018.

58. BREDA, Adriana; FONT, Vicenç; LIMA, Valderez Marina do Rosário; VILLELA PEREIRA, Marcos. Componentes e indicadores de los criterios de idoneidad didáctica desde la perspectiva del Enfoque ontosemiótico. Transformación, v. 14, n. 2, p. 162-176, 2018.

59. FERNANDES, José Antonio. Componentes e indicadores de idoneidade didática de um curso de licenciatura em Matemática: Um levantamento relacionado aos aspectos ecológicos. In: SERNA, Luis Arturo; PÁGES, Daniela (Eds.). ALME: Acta Latinoamericana de Matemática Educativa. Ciudad de México: Comité Latinoamericano de Matemática Educativa, 2018, v. 31, n. 1, p. 1733-1739.

60. GIACOMONE, Belén; GODINO, Juan D.; BELTRÁN-PELLICER, Pablo. Developing the prospective 
Mathematics teachers' didactical suitability analysis competence. Educação e Pesquisa, v. 44, 2018. DOI: 10.1590/s1678-4634201844172011

61. GIACOMONE, Belén; GODINO, Juan D.; WILHELMI, Miguel R.; BLANCO, Teresa F. Developing the onto-semiotic analysis competence of prospective Mathematics teachers. Revista Complutense de Educación, v. 29, n. 4, 1109-1131, 2018.

62. GODINO, Juan D.; GIACOMONE, Belén; FONT, Vicenç; PINO-FAN, Luis R. Conocimientos profesionales en el diseño y gestión de una clase sobre semejanza de triángulos. Análisis con herramientas del modelo CCDM. AIEM, n. 13, p. 63-83, 2018.

63. MONJE, Yerka; SECKEL, María José; BREDA, Adriana. Tratamiento de la inecuación en el currículum y textos escolares chilenos. Bolema: Boletim de Educação Matemática, v. 32, n. 61, p. 480-502, 2018.

64. MOREIRA, Celma Bento; GUSMÃO, Tânia Cristina S.; FONT, Vicenç. Tarefas matemáticas para 0 desenvolvimento da percepção de espaço na educação infantil: Potencialidades y limites. Bolema: Boletim de Educação Matemática, v. 32, n. 60, p. 231-254, 2018.

65. RUZ, Felipe; CONTRERAS, José Miguel; MOLINA-PORTILLO, Elena; GODINO, Juan D. Idoneidad epistémica de programas formativos sobre Didáctica de la Estadística. In: RODRÍGUEZ-MUÑIZ, Luis J.; MUÑIZ-RODRÍGUEZ, Laura; AGUILAR-GONZÁLEZ, Álvaro; ALONSO, Pedro; GARCÍA-GARCÍA, Francisco Javier; BRUNO, Alicia (Eds.). Investigación en Educación Matemática XXII. Gijón: SEIEM, 2018, p. 515-524

66. CARVAJAL, Silvia; GIMÉNEZ, Joaquim; FONT, Vicenç; BREDA, Adriana. La competencia digital en futuros profesores de Matemáticas. In: BADILLO JIMÉNEZ, Edelmira; CLIMENT RODRÍGUEZ, Nuria; FERNÁNDEZ-VERDÚ, Ceneida; GONZÁLEZ ASTUDILLO, María Teresa (Eds.). Investigación sobre el profesor de Matemáticas: Práctica de aula, conocimiento y desarrollo profesional. Universidad de Salamanca, 2019, p. 285-306.

67. GEA María M.; BATANERO, Carmen; ARTEAGA, Pedro; ESTEPA, Antonio. La componente cognitiva del conocimiento didáctico-matemático de profesores en formación sobre correlación y regresión. Caminhos da Educação Matemática, v. 9, n. 2, p. 79-101, 2019.

68. GONÇALVES, Gabriela Maria; FERNANDES, José António. Metodologia trabalho de projeto no ensino de testes de hipóteses: Uma análise da idoneidade didática. REVEMAT: Revista Eletrônica de Educação Matemática, v. 14, n. 2, p. 1-20, 2019.

69. HERRERA-GARCÍA, Karina; DÁVILA-ARAIZA, Teresa; GIACOMONE, Belén; BELTRÁN-PELLICER, Pablo. Diseño de una secuencia didáctica sobre variación lineal dirigida a futuros profesores de Matemáticas de secundaria. In: ROSA-MENDOZA, Alejandro Miguel (Ed.), $5^{\circ}$ Congreso Internacional de Matemática Educativa. Ciudad de México: Lectorum, 2019, p. 32.

70. HUMMES, Viviane Beatriz; FONT, Vicenç; BREDA, Adriana. Uso combinado del estudio de clases y la idoneidad didáctica para el desarrollo de la reflexión sobre la propia práctica en la formación de profesores de Matemáticas. Acta Scientiae, v. 21, n. 1, p. 64-82, 2019.

71. MONTEIRO DE VASCONCELOS, Danilo; CARVALHO, José Ivanildo Felisberto de. Idoneidade 
cognitivo-afetiva de uma sequência didática para a construção do conceito de razões trigonométricas por meio de uma história em quadrinhos. Revista de Educação Matemática e Tecnológica Iberoamericana, v. 10, n. 2, 1-24, 2019.

72. MORALES-LÓPEZ, Yuri; FONT, Vicenç. Valoración realizada por una profesora de la idoneidad de su clase de Matemáticas. Educação e Pesquisa, v. 45, 2019.

73. RUZ, Felipe; MOLINA-PORTILLO, Elena; GARCÍA, J. M. C. Guía de valorización de la idoneidad didáctica de procesos de instrucción en Didáctica de la Estadística. Bolema: Boletim de Educação Matemática, v. 33, n. 63, p. 135-154, 2019.

74. BELTRÁN-PELLICER, Pablo; GIACOMONE, Belén; BAGUÉ, NURIA. Propuestas de aplicación de indicadores de idoneidad didáctica en Probabilidad y Estadística: Análisis de vídeos educativos. In: RIBEIRO CAMPOS, Celso; PAVAN PERIN, Andréa (Orgs.), Investigações hispano-brasileiras em educação estatística. Brasil: Akademy, 2020, p. 54-60.

75. BREDA, Adriana. Características del análisis didáctico realizado por profesores para justificar la mejora en la enseñanza de las Matemáticas. Bolema: Boletim de Educação Matemática, v. 34, n. 66, p. 69-88, 2020.

76. BURGOS, María; BELTRÁN-PELLICER, Pablo; GODINO, Juan D. La cuestión de la idoneidad de los vídeos educativos de Matemáticas: Una experiencia de análisis con futuros maestros de educación primaria. Revista Española de Pedagogía, v. 78, n. 275, p. 27-49, 2020.

77. BURGOS, MARÍA; CASTILLO, María José; BELTRÁN-PELLICER, Pablo; GIACOMONE, Belén; GODINO, Juan D. Análisis didáctico de una lección sobre proporcionalidad en un libro de texto de primaria con herramientas del Enfoque ontosemiótico. Bolema: Boletim de Educação Matemática, v. 34, n. 66, p. 40-68, 2020.

78. BURGOS, María; CASTILLO, María José; GODINO, Juan D. Formación de profesores de Matemáticas en el análisis de libros de texto. In: BALDA ÁLVAREZ, Paola; PARRA ZAPATA, Mónica Marcela; SOSTENES GONZÁLEZ, Horacio Saúl (Eds.). ALME: Acta Latinoamericana de Matemática Educativa. Ciudad de México: Comité Latinoamericano de Matemática Educativa, 2020, v. 33, n. 1, p. 534-546.

79. GEA MARÍA M.; BATANERO, CARMEN; Estrada, A. Evaluación de la idoneidad afectiva del trabajo en proyectos estadísticos por profesores en formación. In: BALDA ÁLVAREZ, Paola; PARRA ZAPATA, Mónica Marcela; SOSTENES GONZÁLEZ, Horacio Saúl (Eds.). ALME: Acta Latinoamericana de Matemática Educativa. Ciudad de México: Comité Latinoamericano de Matemática Educativa, 2020, v. 33 , n. 1, p. 513-522.

80. HUMMES, Viviane Beatriz; BREDA, Adriana; SECKEL, María José; FONT, Vicenç. Criterios de idoneidad didáctica en una clase basada en el lesson study. Praxis \& Saber, v. 11, n. 26, e-0667, 2020. DOI: 10.19053/22160159.v11.n26.2020.10667

81. MORALES-LÓPEZ, Yuri; ARAYA-ROMÁN, Daniela. Apoyando a los futuros profesores a reflexionar. Acta Scientiae, v. 22, n. 1, p. 88-112, 2020.

82. PINO-FAN, Luis R.; BÁEZ-HUAIQUIÁN, Daniela Ilse; MOLINA-CABERO, Juan G.; HERNÁNDEZ- 
ARREDONDO, Elizabeth. Criterios utilizados por profesores de Matemáticas para el planteamiento de problemas en el aula. Uniciencia, v. 34, n. 2, p. 114-136, 2020.

83. RUZ, Felipe; MOLINA-PORTILLO, Elena; CONTRERAS, José Miguel. Idoneidad didáctica de procesos de instrucción programados sobre Didáctica de la Estadística. PNA, v. 14, n. 2, 141-172, 2020.

84. SECKEL, María José; FONT, Vicenç. Competencia reflexiva en formadores del profesorado de Matemática. MAGIS, Revista Internacional de Investigación en Educación, v. 12, n. 25, p. 127-144, 2020.

85. TORRES, C. Developing teachers' didactic analysis competence by means of a problem-posing strategy and the quality of posed mathematical problems. In: K. O. VILLALBA-CONDORI, A. ADURÍZBRAVO, J. LAVONEN, L-H. WANG Y T-H. WANG (Eds.). Education and Technology in Sciences. First International Congress, CISETC 2019, Arequipa, Peru, December 10-12, 2019, Revised selected papers (pp. 88-100). Cham: Springer, 2020.

Otros resultados fueron excluidos de la muestra por falta de accesibilidad o bien, por no ser publicaciones online.

A partir de la revisión, análisis crítico e interpretación de los 85 documentos, como segundo resultado se determinó un sistema de categorías emergentes que permite clasificar los contextos de uso en los cuales se aplica la Idoneidad Didáctica, es decir, los problemas de investigación que se pueden afrontar.

Ese sistema contempla nueve categorías emergentes:

- La Idoneidad Didáctica como herramienta para valorar orientaciones curriculares, planes de estudio o programas de formación.

- La Idoneidad Didáctica como herramienta para valorar libros de texto.

- La Idoneidad Didáctica como herramienta para valorar videos educativos.

- La Idoneidad Didáctica como herramienta para determinar criterios de idoneidad de tópicos específicos.

- La Idoneidad Didáctica como herramienta para desarrollar la competencia de análisis didáctico en la formación docente.

- La Idoneidad Didáctica como herramienta para valorar el diseño y/o la implementación de un ciclo educativo.

- La Idoneidad Didáctica como herramienta para valorar las acciones didácticas 0 producciones escritas de los profesores en formación. 
- La Idoneidad Didáctica como herramienta para interpretar, en sus términos, valoraciones realizadas por profesores o futuros profesores.

- La Idoneidad Didáctica como herramienta para diseñar actividades y secuencias didácticas a partir de los criterios de idoneidad.

En función de la interpretación que hacen los autores del artículo a la luz del conocimiento sobre la Idoneidad Didáctica, muchos de los trabajos fueron ubicados simultáneamente en diversas categorías emergentes, sea porque los investigadores persiguen propósitos múltiples, sea porque en la investigación intervienen distintos actores que hacen usos diferenciados de la herramienta; es el caso, por ejemplo, de aquellos trabajos en los que los docentes en formación desarrollan la competencia de análisis didáctico, y a la vez, el formador/investigador, hace uso de la herramienta para valorar el diseño y/o la implementación del ciclo formativo implementado, proponiendo mejoras. En la Tabla 2 se han clasificado los 85 trabajos analizados en función de las categorías emergentes a las que pertenecen.

Tabla 2: Clasificación de los trabajos en función de las categorías emergentes

\begin{tabular}{|c|c|c|}
\hline $\begin{array}{l}\text { Categorías emergentes } \\
\text { La Idoneidad Didáctica como herramienta para... }\end{array}$ & $\begin{array}{c}\text { Cantidad de } \\
\text { trabajos }\end{array}$ & Trabajos* \\
\hline $\begin{array}{l}\text { valorar orientaciones curriculares, planes de estudio } \\
\text { o programas de formación }\end{array}$ & 8 & $15,21,22,53,59,63,65,83$ \\
\hline valorar libros de texto & 6 & $22,38,50,63,77,78$ \\
\hline valorar videos educativos & 3 & $55,74,76$ \\
\hline $\begin{array}{l}\text { determinar criterios de idoneidad didáctica de un } \\
\text { tópico específico }\end{array}$ & 10 & $3,15,21,42,47,48,51,56,66,73$ \\
\hline $\begin{array}{l}\text { desarrollar la competencia de análisis didáctico en la } \\
\text { formación docente }\end{array}$ & 30 & $\begin{array}{l}4,5,6,10,12,14,16,18,19,20,24,29 \\
30,31,34,40,43,52,54,60,61,62,67 \\
70,76,78,79,81,84,85\end{array}$ \\
\hline $\begin{array}{l}\text { valorar el diseño y/o la implementación de un ciclo } \\
\text { educativo }\end{array}$ & 48 & $\begin{array}{l}1,2,4,5,6,8,9,10,11,12,13,14,17 \\
20,23,24,25,26,27,30,31,32,33,35 \\
39,43,44,45,49,52,54,56,57,59,60 \\
61,62,64,67,68,69,70,71,72,75,79 \\
81,84\end{array}$ \\
\hline $\begin{array}{l}\text { valorar las acciones didácticas o producciones } \\
\text { escritas de los profesores en formación }\end{array}$ & 4 & $25,41,66,85$ \\
\hline $\begin{array}{l}\text { interpretar, en términos de la idoneidad didáctica, } \\
\text { valoraciones realizadas por profesores o futuros } \\
\text { profesores }\end{array}$ & 10 & $7,25,36,37,46,58,72,75,80,82$ \\
\hline $\begin{array}{l}\text { diseñar actividades y secuencias didácticas a partir } \\
\text { de los criterios de idoneidad didáctica }\end{array}$ & 5 & $20,28,40,49,64$ \\
\hline
\end{tabular}


Tal como se visualiza en la Tabla 2, las dos categorías que reúnen la mayor cantidad de trabajos (48 y 30 , respectivamente) son las que remiten al uso de la Idoneidad Didáctica para valorar el diseño y/o la implementación de un ciclo educativo, y para desarrollar la competencia análisis didáctico en la formación docente.

Como se puede observar, son categorías que caracterizan a la Idoneidad Didáctica como herramienta metodológica para abordar la optimización de los procesos instruccionales, utilizada no solo por investigadores, sino también por profesores y estudiantes en formación inicial y continua, revelándose como un tema que interesa a toda la comunidad científica.

Si se tiene en cuenta que los trabajos 1 a 7 fueron publicados entre 2005 y 2008 , los que tienen número de orden 8 a 17, entre 2009 y 2012, los que tienen número de orden 18 a 41, entre 2013 y 2016, y los que tienen número de orden 42 a 85, entre 2017 y 2020, se advierte que cuatro de las nueve categorías emergentes (la idoneidad didáctica como herramienta para valorar libros de texto, valorar videos educativos, valorar acciones didácticas o producciones escritas de profesores en formación, y diseñar actividades y secuencias didácticas a partir de los criterios de idoneidad) solo contienen trabajos publicados de 2013 en adelante. Es más, todos los trabajos de la categoría que hace referencia a la valoración de videos educativos fueron publicados recién durante el último cuatrienio.

Así como la lectura en clave cronológica de la Tabla 1 muestra la expansión cuantitativa de cuatrienio en cuatrienio de las investigaciones que utilizan como herramienta a la Idoneidad Didáctica, la lectura de la Tabla 2 en esa misma clave, muestra una expansión cualitativa, en términos de la aparición de nuevos y promisorios contextos de uso.

Para finalizar, a continuación se discuten algunos resultados derivados del análisis de las categorías a priori que permiten recuperar el conocimiento acumulado y generar nuevos conocimientos, comprensiones y futuras líneas de investigación.

- Nivel educativo

Desde el punto de vista del nivel educativo, tres investigaciones refieren a la Educación Infantil: 3, 49 y 64; una, a la Educación Básica: 27; 10, a la Educación Primaria: 11, 23, 38, 39, 45 , 47, 48, 51, 77 y 80; 12, a la Educación Secundaria: 8, 9, 13, 22, 35, 44, 50, 55, 56, 57, 71 y 72; una, a la Educación Primaria y Secundaria: 63; ocho se focalizan en el nivel universitario: 1, 2, 7 , 17, 28, 32, 42 y 68 . Todos los demás trabajos se ocupan de la formación docente.

Con respecto a las 30 publicaciones en las que la idoneidad didáctica se emplea como herramienta para desarrollar la competencia de análisis didáctico en la formación docente, 24 de 
ellas refieren a la formación inicial (sea de profesores de Educación Primaria, sea de profesores de Matemática para la Educación Secundaria); las seis investigaciones restantes versan sobre el desarrollo de dicha competencia en la formación continua: tres, en la formación a nivel de máster (publicaciones 18, 54 y 70); dos, en la formación de formadores (publicaciones 40 y 84); una, en la formación de docentes en servicio (publicación 85).

\section{- Facetas}

De los 81 trabajos que explicitan qué facetas de la idoneidad didáctica abordan, 22 valoran una única faceta (publicaciones 9, 12, 15, 18, 19, 22, 34, 43, 44, 47, 48, 50, 55, 57, 59, 63, 65, 67, $74,75,76$ y 79$)$, seis valoran dos facetas $(13,16,24,42,45$ y 71$)$, tres, tres facetas (1, 23 y 68), tres, cuatro facetas $(41,52$ y 77$)$, dos, cinco facetas (7 y 78$)$ y 45 , las seis facetas $(2,4,5,6,8,10$, $11,14,17,20,21,25,26,27,28,29,30,31,32,33,35,36,37,38,39,40,46,49,51,53,54,56$, $58,60,61,62,64,66,70,72,73,80,81,83$ y 84$)$.

\section{- Contenido}

El contenido es explicitado por 59 de las 85 investigaciones. Los contenidos abordados son: noción de función (publicaciones 1, 2 y 50), estocástica, intuiciones sobre el azar, estadística, estadística elemental, nociones probabilísticas o estadísticas, contenidos probabilísticos, probabilidad $(4,5,6,12,15,26,44,54,56,73$ y 83$)$, poliedro regular (8), proporcionalidad y repartos proporcionales $(9,20,35,55,76,77$ y 78$)$, ecuaciones, inecuaciones y resolución de ecuaciones (11, 20, 31 y 63), límite (13 y 52), operaciones numéricas básicas (16 y 80), derivada (17 y 22), geometría esférica (18), funciones trigonométricas (18), integrales, integral de Riemann, cálculo integral, teorema fundamental del cálculo (19, 28, 37 y 46), mediatriz (20), teorema de Thales (20), semejanza de triángulos (20,60 y 62), circunferencia y círculo (23), análisis de datos (24 y 43), caída libre (Física, 32), polinomios (36), gráficos estadísticos (38), patrones de medida no convencionales (45), percepción y visualización del espacio, geometría espacial (47, 48, 49 y 64), masa (51), matemática financiera (58), correlación y regresión (67 y 79), pruebas de hipótesis (68), variación lineal (69), razones trigonométricas (71), función logarítmica (72 y 81), mediana (74).

- Difusión internacional

La expansión cuantitativa y cualitativa ya mencionada tiene su correlato en la difusión internacional de las investigaciones que aplican la noción, tal como muestra la Figura 2, en la que se han georreferenciado las investigaciones según el país en el que fueron publicadas. 
Figura 2. Cantidad de trabajos publicados por país de edición

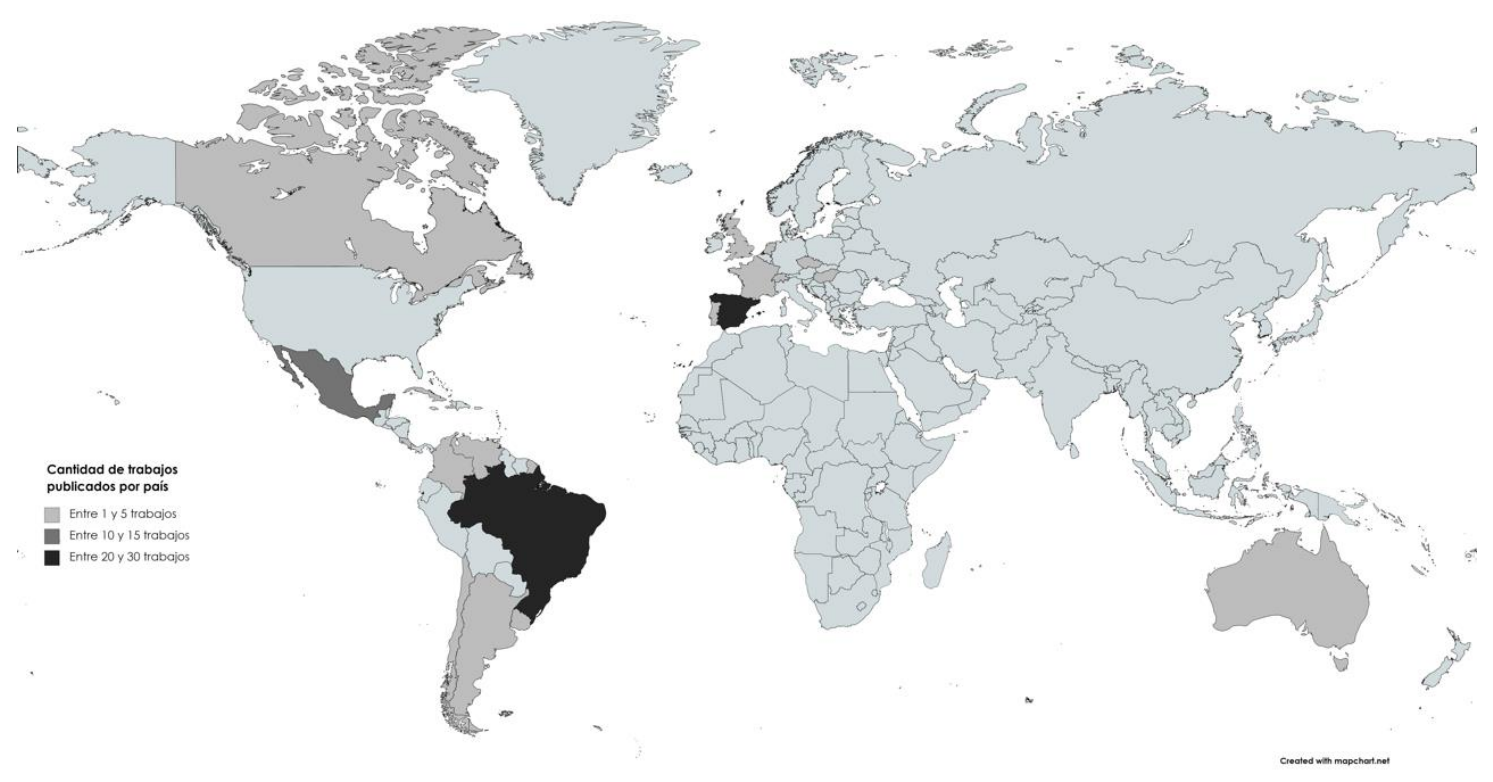

Fuente: Elaboración propia (mapa creado con mapchart.net) (2021).

Todos estos resultados ratifican la contemporaneidad y la vigencia de la noción de idoneidad, y el interés que los investigadores invierten en ella.

\section{Conclusiones}

Este estudio documental es una contribución a la construcción del estado del arte sobre el constructo Idoneidad Didáctica desarrollado en el marco del EOS.

El único antecedente significativo que se ha encontrado en esta misma línea es el trabajo de Breda, Font y Lima (2015). Los autores analizaron y categorizaron 30 trabajos publicados entre 2006 y 2015, referidos, fundamental pero no exclusivamente, a los usos de la Idoneidad Didáctica en la formación de profesores de diferentes niveles educativos en cinco países: Argentina, Brasil, Chile, España y México. 23 de los 30 trabajos incluidos en Breda et al. (2015) también forman parte de la muestra de material empírico que sustenta a este artículo, pero fueron analizados desde otra perspectiva metodológica; en efecto, las categorías emergentes que aquí se identifican no se restringen al uso de la Idoneidad Didáctica en la formación docente, sino que dan cuenta de múltiples contextos de empleo y de diversos objetos de estudio.

Los resultados ponen de manifiesto la expansión de la herramienta, tanto desde el punto de vista cuantitativo, por la creciente cantidad de investigaciones que la aplican, como desde el punto de vista cualitativo, por el surgimiento progresivo de nuevos contextos de uso. 
El resultado principal es el sistema de nueve categorías emergentes; lo es por su potencial utilidad para los investigadores, sea para categorizar sus trabajos, sea para dirigir sus investigaciones hacia espacios de intersección entre categorías, sea, incluso, para detectar lagunas o nichos de vacancia en la investigación. En este sentido, la información que ofrece la Tabla 2 constituye un aporte significativo, ya que provee ejemplos que validan cada categoría.

Un punto particularmente interesante es que la Idoneidad Didáctica no se muestra como una herramienta cuyo uso solo está al alcance de los investigadores; las investigaciones muestran que también los profesores pueden usarla competentemente, sea para proponer mejoras justificadas a su práctica profesional, para seleccionar videos educativos o libros de texto con criterios científicos, o para valorar las producciones de sus alumnos, tareas específicas 0 incidentes didácticos puntuales.

Una categoría que merece especial atención es la que remite al empleo de la Idoneidad Didáctica como herramienta para desarrollar la competencia de análisis didáctico en la formación docente. Las investigaciones que se inscriben en esta categoría muestran una evolución en el uso del constructo: de consignas generales para el desarrollo de la competencia de análisis didáctico, hacia consignas más específicas referidas al desarrollo de la competencia de análisis de la idoneidad didáctica; este cambio se produce a partir de 2017, año en el cual Godino et al. (2017) desarrollan el modelo CCDM.

El análisis en función de las categorías definidas a priori permite concluir que la mayoría de los trabajos tienden a analizar contenidos matemáticos sobre estadística y probabilidad, funciones, geometría y proporcionalidad. Mientras que en poco más de la mitad de los trabajos se analizan las idoneidades de las seis facetas que interaccionan en un proceso de estudio, en los restantes se hace foco en algunas de ellas. Los trabajos de la categoría que utiliza la Idoneidad Didáctica para valorar un ciclo educativo se refieren en general a procesos de estudio breves, por ejemplo, valoración de una secuencia didáctica. Solo 3 casos valoran un curso completo (1 año, 1 semestre, 3 años). En cuanto al nivel educativo, exceptuando las investigaciones que indagan en la formación docente, prevalecen las que inciden en los niveles primario y secundario.

Sin duda, estos resultados, junto con las categorías emergentes, marcan claras tendencias en las preocupaciones de la comunidad educativa reflejando una visión general sobre las temáticas de interés. 
Reconocimiento.

Trabajo realizado en el marco de los proyectos PGC2018-098603-B-I00 (MCIU/AEI/FEDER, UE), PFID-FID-2021-45 (Panamá) y Grupo S60_20R - Investigación en Educación Matemática (Gobierno de Aragón y Fondo Social Europeo).

\section{Referencias}

BARDIN, Laurence. Análisis de contenido (2da ed.). Madrid: Ediciones Akal, 1996.

BELTRÁN-PELLICER, Pablo. Evaluación de la idoneidad didáctica de una experiencia de enseñanza del azar y probabilidad en tercer curso ESO. 2016. 126f. Tesis de Fin de Máster. Universidad de Granada, Granada, España.

BREDA, Adriana. Melhorias no ensino de matemática na concepção de professores que realizam o mestrado profmat no rio grande do sul: uma análise dos trabalhos de conclusão de curso. 2016. 335f. Tesis doctoral. Pontifícia Universidade Católica do Rio Grande do Sul, Brasil.

BREDA, Adriana; FONT, Vicenç; LIMA, Valderez Marina do Rosário. A noção de idoneidade didática e seu uso na formação de professores de matemática. Jornal Internacional de Estudos em Educação Matemática, v. 8, n. 2, p. 1-41, 2015.

BREDA, Adriana; FONT, Vicenç; PINO-FAN, Luis R. Criterios valorativos y normativos en la Didáctica de las Matemáticas: el caso del constructo idoneidad didáctica. Bolema: Boletim de Educação Matemática, v. 32, n. 60, p. 255-278, 2018. DOI: 10.1590/1980-4415v32n60a13

BURGOS, María. Niveles de alegorización en el razonamiento proporcional desde las perspectivas institucional y personal. Implicaciones para la formación de profesores de matemáticas. 2020. 399f. Tesis doctoral. Universidad de Granada, Granada, España.

CRISÓSTOMO, Edson. Idoneidad de procesos de estudio del cálculo integral en la formación de profesores de matemáticas: Una aproximación desde la investigación en didáctica del cálculo y el conocimiento profesional. 2012. 471f. Tesis doctoral. Universidad de Granada, Granada, España.

FONT, Vicenç. Competencias profesionales en la formación inicial de profesores de Matemáticas de secundaria. Unión, n. 26, p. 9-25, 2011.

GIACOMONE, Belén. Desarrollo de competencias y conocimientos didáctico-matemáticos de futuros profesores de educación secundaria en el marco del enfoque ontosemiótico. 2018. 265f. Tesis doctoral. Universidad de Granada, Granada, España.

GODINO, Juan D. Un enfoque ontológico y semiótico de la cognición matemática. Recherches en Didactiques des Mathematiques, v. 22, n. 2/3, p. 237-284, 2002.

GODINO, Juan D. Indicadores de la idoneidad didáctica de procesos de enseñanza y aprendizaje de las matemáticas. Cuadernos de Investigación y Formación en Educación Matemática, $n$. 11, p. 111-132, 2013. 
GODINO, Juan D.; BATANERO, Carmen; FONT, Vicenç. The onto-semiotic approach to research in mathematics education. ZDM Mathematics Education, n. 39, p. 127-135, 2007. DOI: 10.1007/s11858-006-0004-1

GODINO, Juan D.; BATANERO, Carmen; FONT, Vicenç. El enfoque ontosemiótico: implicaciones sobre el carácter prescriptivo de la didáctica. Revista Chilena de Educación Matemática, v. 12, n. 2, p. 3-15, 2020. DOI: 10.46219/rechiem.v12i2.25

GODINO, Juan D.; BURGOS, María; GEA, María Magdalena. Analyzing theories of meaning in mathematics education from the onto-semiotic approach. International Journal of Mathematical Education in Science and Technology, 2021. DOI: 10.1080/0020739X.2021.1896042

GODINO, Juan D. et al. Enfoque Ontosemiótico de los Conocimientos y Competencias del Profesor de Matemáticas. Bolema: Boletim de Educação Matemática, v. 31, n. 57, p. 90-113, 2017. DOI: $10.1590 / 1980-4415 v 31 n 57 a 05$

HOYOS BOTERO, Consuelo. Un modelo para investigación documental: guía teórico-práctica sobre construcción de Estados del Arte con importantes reflexiones sobre la investigación. Medellín: Señal Editora, 2000.

PATTON, Michael Quinn. Qualitative research \& evaluation methods (3ra ed.). Thousand Oaks: Sage, 2002.

RIVAS, Hernán. Idoneidad didáctica de procesos de formación estadística de profesores de educación primaria. 2014. 404f. Tesis doctoral. Universidad de Granada, Granada, España.

VASILACHIS DE GIALDINO, Irene. La investigación cualitativa. In: Estrategias de investigación cualitativa. Barcelona: Gedisa, 2006, p. 23-64. 